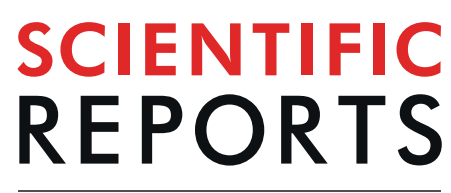

natureresearch

Check for updates

\title{
Mechanomyographic Measures of Muscle Contractile Properties are Influenced by Electrode Size and Stimulation Pulse Duration
}

\author{
Francisco Piqueras-Sanchiz ${ }^{1}$, Saúl Martín-Rodríguez ${ }^{2}$, Fernando Pareja-Blanco ${ }^{1}$, \\ Luis Baraja-Vegas ${ }^{3}$, Jorge Blázquez-Fernández ${ }^{4}$, Iker J. Bautista ${ }^{4}$ \& Óscar García-García ${ }^{5 凶}$
}

The aim was to determine the effects of changing pulse duration and electrode size on muscle contractile properties. Thirty-six healthy young male participated in the study (age $24.8 \pm 5.8$ years; height $178.2 \pm 0.6 \mathrm{~cm}$; body mass $71.8 \pm 7.3 \mathrm{~kg}$; self-reported weekly moderate intensity activity $3.5 \pm 1.2 \mathrm{~h} \cdot$ week $^{-1}$ ). Tensiomyography was used to assess rectus femoris (RF) and vastus medialis (VM) muscles neuromuscular properties of the dominant leg according to the electrode size $(3.2-5 \mathrm{~cm})$ and the stimulus length $(0.2,0.5$, and $1 \mathrm{~ms})$. Maximal radial displacement $(\mathrm{Dm})$; Contraction time (Tc); Delay time (Td); Sustained time (Ts) and Half relaxation time (Tr) were measured. Relative and absolute reliability was quantified. To analyze the effects of the electrode and the stimulus length, a repeated-measures analysis of variance was used. Dm and Tc parameters showed for both muscles an excellent relative (0.95-0.99) and absolute reliability (1.6-4.2\%). However, Ts and Tr showed low values of absolute reliability (4.4-40.9\%). The duration of the stimulus length applied to the RF and VM and electrode size significantly influences muscle's contractile properties $\left(p<0.05 ; \eta^{2}=0.09-0.60\right)$. The Dm increases substantially as the duration of the stimulus increases and with the use of the larger electrode in both muscles. However, Tc and Td are less affected by both conditions and not entirely clear. Practically, our study suggests that a stimulus pulse duration of $1 \mathrm{~ms}$ together with a $5 \times 5 \mathrm{~cm}$ electrode is necessary to reach a reliable and reproducible assessment of both RF and VM muscles contractile properties.

Mechanomyography (MMG) is a set of different methods to record mechanical properties, such as muscle's belly displacement and contraction time (Tc), in response to either voluntary or electrically stimulated muscle contraction $^{1}$. Among the different MMG methods as vibromyography or sonomyography, tensiomyography (TMG) has received great attention in the last decade by the scientific and clinical community ${ }^{2}$. This method has been utilized in a variety of applications, including estimating myosin heavy chain composition ${ }^{3}$, determining muscle fiber type populations $s^{4,5}$, detecting stiffness or atroph ${ }^{6,7}$, assessing athlete's performance ${ }^{8}$, evaluating muscle fatigue in laboratory or field conditions ${ }^{2}$ or identifying muscle dysfunctions and treatment-effects ${ }^{9,10}$, among other functions.

TMG is one of the MMG techniques that records muscle contractile properties in response to electrically stimulated muscle contractions, which are evoked through the delivery of transcutaneous neuromuscular stimulations (TNS). Most of TMG protocols determine the maximal TNS with a 'current ramp' protocol. This methodology entails delivering a series of incremental TNS impulses by increasing amperage (mA) whilst keeping a constant voltage and stimulus length until a maximal muscle contraction is reached - as determined by maximal radial displacement $(\mathrm{Dm})$. Little attention has received in the literature one of the aforementioned variables (i.e. stimulus length) of the TNS protocol. The stimulus length, i.e. the duration of the applied TNS stimulation, influences the magnitude of the electrical energy delivered to the muscle. Thus, stimulus length influences the amplitude and timing of the TMG waveforms. Unfortunately, a lack of a standardized protocol has led to a variety

${ }^{1}$ Physical Performance \& Sports Research Center, Universidad Pablo de Olavide, Seville, Spain. ${ }^{2}$ Department of Physical Education, University of Las Palmas de Gran Canaria, Las Palmas de Gran Canaria, Spain. ${ }^{3}$ Department of Physiotherapy, Catholic University of Valencia, Valencia, Spain. ${ }^{4}$ Faculty of Physiotherapy and Podology, Catholic University of Valencia, Valencia, Spain. ${ }^{5}$ Laboratory of Sports Performance, Physical Condition and Wellness, Faculty

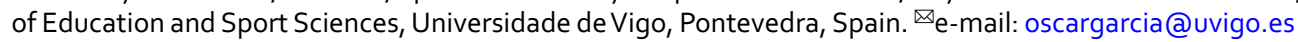


of TNS stimulus length being reported in humans ranging from $200 \mu$ s to $1000 \mu$ s in different muscles ${ }^{11-13}$. To our knowledge, one of the few studies that have addressed this question on MMG was published more than 10 years ago $^{14}$. These authors tested a wide amount of stimulus length ranging from 50 to $500 \mu$ s, at $50-\mu$ s increments. They showed that the contractile properties of the muscle were considered stable at pulse durations above $300 \mu \mathrm{s}$, which was similar with previously published data for the same muscle ${ }^{14}$. Notably, these authors used a laser MMG sensor while TMG uses a contact-displacement sensor (CDS). In this regard, both laser and CDS offer good-to-excellent reliability, although significant systematic bias was identified with the CDS recording higher mean values ${ }^{15}$. However, it was previously identified that these differences may not be considered clinically significant. Despite the above, these authors also found that the wide limits of agreement $(-19.0 \mathrm{~ms}$ and $25.2 \mathrm{~ms})$ identified between half-relaxation time ( $\mathrm{Tr}$ ) (i.e., the time taken from 90 to $50 \%$ of $\mathrm{Dm}$ ) measures, were considered unreliable from a clinical perspective ${ }^{15}$. This finding is consistent with data from a contemporary review on the reliability and measurement error of TMG, which concluded that Tr should not be used for clinical or research purposes ${ }^{16}$.

Inter-electrode distance (IED) for electrical stimulation is another key factor in the measurements of any MMG device. This parameter has been examined in some studies with several muscles, which have described that IED significantly influences TMG waveform, thus negatively affecting the measure ${ }^{17-19}$. Despite having studied IED little, the effect of the electrode size on the evoked response has been hardly studied in MMG devices, and only in the field of physical therapy to analyse the thresholds of sensory and motor excitation ${ }^{20,21}$. These studies observed that larger electrodes required greater voltage output but less pulse-charge density than the smaller electrodes. The above, transferred to TMG, means that electrode size could influence the TMG waveform, thus exhibiting different responses of the contractile properties depending on the size.

Therefore, it is important to increase the accuracy of TMG assessments while minimizing the measurement error to reach reliable data and compare between studies. It is also important for practitioners for the same issue, so they can be able to accurately and objectively compare several intra- and inter-subjects measurements over time.

We hypothesised that the larger the electrode size and stimulus pulse duration allowed by TMG, the greater the data accuracy and the less the measurement error. Therefore, the aims of this study were: 1) to determine the effect of changing the pulse duration on muscle contractile properties and 2) to discriminate if changing electrode size with different pulse duration affects muscle contractile properties.

\section{Methods}

Participants. Thirty-six healthy young and moderately active male volunteers (age $24.8 \pm 5.8$ years; height $178.2 \pm 0.6 \mathrm{~cm}$; body mass $71.8 \pm 7.3 \mathrm{~kg}$; self-reported weekly moderate intensity activity $3.5 \pm 1.2 \mathrm{~h} \cdot \mathrm{week}^{-1}$ ) who had not suffered muscle or tendon injuries in the previous 6 months participated in the study.

The sample size was calculated for each evaluated muscle using the G*POWER software (Heinrich-Heine-Universität Düsseldorf. Germany). The results have showed that for the Dm of the rectus femoris (RF) and vastus medialis (VM), with an alpha of 0.05 , a statistical power of 0.80 and an effect size of 0.25 , at least 28 and 36 participants were needed respectively. Therefore, a sample size of 36 participants was selected.

Compliance with ethical standards. All voluntary participants were informed of the research objectives and had the possibility to withdraw at any time from the investigation without any penalty. Informed consent was obtained from all individual participants included in the study. The study was conducted during 4 weeks, according to the Declaration of Helsinki, and the protocol was fully approved by the Ethics Committee of the Catholic University of Valencia (UCV2017-2018-73). The authors declare that they have no conflict of interest.

Experimental design. A descriptive cross-sectional design was used in order to analyse the effect of the electrode size and the stimulus duration on the parameters obtained with TMG. All participants measurements were made on the same day, in the same room and under the same temperature and humidity conditions $\left(23.3 \pm 0.6^{\circ} \mathrm{C}\right.$ and $45 \pm 6.6 \%$ respectively) measured with a weather station using an external sensor. The volunteers were instructed to refrain from moderate or heavy physical activities within $72 \mathrm{~h}$ prior the assessments.

Before the data collection, participants were familiar with the electrostimulation stimulus. A 10 min period was established lying face up on a stretcher in order to obtain a muscular relaxation state. All subjects were shaved and the evaluated muscle area was cleaned with alcohol to favour impedance. This was done both in the familiarisation and in the measurement period. New electrodes were used in each measurement using 3.2 or $5 \mathrm{~cm}$ self-adhesive circular electrodes. Different measurements were established in the VM and the RF muscles of the dominant leg according to the electrode size $(3.2-5 \mathrm{~cm})$ and the stimulus length $(0.2,0.5$, and $1 \mathrm{~ms})$, so that 36 measurements were obtained in each participant, 18 in the RF and 18 in the VM. The total duration of the evaluation was $\pm 60 \mathrm{~min}$. The intervention conditions were randomised (see Table 1), that is, the muscle evaluation order, the used electrode sequence and the stimulus length was varied. i.e., subject 1 started in the VM with a $5 \mathrm{~cm}$ electrode and with $1 \mathrm{~ms}$ stimulus length, then $5 \mathrm{~cm}$ electrode being pulse duration $0.2 \mathrm{~ms}$ and finally electrode $5 \mathrm{~cm}$ and stimulus length $0.5 \mathrm{~ms}$.

Contractile properties assessment. TMG was used to assess the neuromuscular properties of both RF and VM muscles of the dominant leg. Measurements were taken under static and relaxed conditions. Prior to performing the measurements, an accurate digital displacement-transducer (GK 40, Panoptik doo, Ljubljana, Slovenia) was perpendicularly positioned at the highest point of the muscle belly. The exact positioning of the electrodes for the RF and VM muscles were in concordance with the recommendations of the SENIAM project ${ }^{22}$. RF electrodes were placed at $50 \%$ on the line from the anterior superior iliac spine to the superior part of the patella and the VM electrodes were placed at $80 \%$ on the line between the anterior superior iliac spine and the joint space in front of the anterior border of the medial ligament. To assure the same electrode placement between 


\begin{tabular}{|c|c|c|c|c|c|c|c|}
\hline \multirow{2}{*}{$\begin{array}{l}\text { Electrode Size } \\
\text { SUBJECT }\end{array}$} & \multicolumn{3}{|c|}{$3.2 \mathrm{~cm}$} & \multirow[b]{2}{*}{ SUBJECT } & \multicolumn{3}{|c|}{$5 \mathrm{~cm}$} \\
\hline & \multicolumn{3}{|c|}{$\begin{array}{l}\text { Blocking } \\
\text { subjects }\end{array}$} & & \multicolumn{3}{|c|}{\begin{tabular}{|l|}
$\begin{array}{l}\text { Blocking } \\
\text { subjects }\end{array}$ \\
\end{tabular}} \\
\hline 1 & A & B & $\mathrm{C}$ & 1 & A & B & C \\
\hline 2 & A & C & B & 2 & A & C & B \\
\hline 3 & $\mathrm{~B}$ & A & $\mathrm{C}$ & 3 & B & A & C \\
\hline 4 & B & C & A & 4 & B & C & A \\
\hline 5 & C & A & B & 5 & C & A & B \\
\hline 6 & $\mathrm{C}$ & B & A & 6 & $\mathrm{C}$ & B & A \\
\hline
\end{tabular}

Table 1. Blocking subjects. A: 0.2 milliseconds (ms); B: $0.5 \mathrm{~ms}$; C: $1 \mathrm{~ms}$.

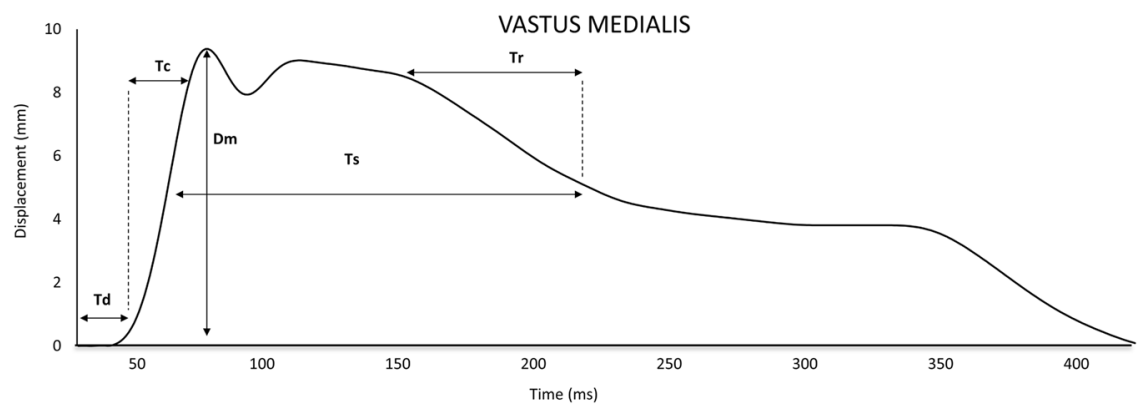

Figure 1. TMG parameters extracted of displacement-time curve of Vastus Medialis.

the consecutive measurements, the point was marked with a dermatological pen. To elicit the twitch responses, two circular self-adhesive electrodes with difference sizes $(3.2$ or $5 \mathrm{~cm}$ ) (Dura-Stick premium, CEFAR-COMPEX, Hannover, Germany) were connected to an electric stimulator (TMG-S1 doo, Ljubljana, Slovenia) and positioned on the muscle surface, following the arrangement of the fibres. Electrodes were placed symmetrically with a IED of approximately $5 \mathrm{~cm}$, placing the positive electrode in the proximal area of the muscle above the measurement point and the negative electrode in the distal area below the measurement point, according to previous investigations ${ }^{23}$. Both RF and VM muscles were measured in the supine position, with the knee joint fixed at a $145^{\circ}$ knee flexion angle, once again by means of a wedge cushion designed for such purpose. The electrical stimulation was applied with different duration of the stimulus $(0.2,0.5$ or $1 \mathrm{~ms}$ ) with a current amplitude of $100 \mathrm{~mA}$ (i.e., single-twitch). Single-twitch is defined as the contractile response to a single electrical impulse and it is a specific type of evoked muscle activity used to characterise the mechanical properties of a muscle or a single motor unit. Although to identify the maximal required stimulus amplitude, and thus the peak muscle response, a progressive incremental approach has been adopted in most studies on the topic ${ }^{2,8}$, using in some of them a single-twitch ${ }^{24-26}$. Typically, studies report that the peak response occurs at stimulus amplitudes between 60 and $100 \mathrm{~mA}$, being in larges muscles, such as the lower limbs, much closer to $100 \mathrm{~mA}^{2}$. Therefore, the decision to use a single-twitch of $100 \mathrm{~mA}$ to homogenize the results derived from the different experimental configurations (i.e., electrode size and stimulation pulse duration). A 60 s rest period was allowed between each electrical stimulus to avoid fatigue or post-tetanic activation while a $120 \mathrm{~s}$ rest period was established between conditions. All measurements were taken by the same experienced evaluator.

In order to ensure the reliability of the TMG assessment, two measurements were taken in each participant in all conditions (stimulus length and electrode size). Between one and two minutes passed between the test-retest of each condition.

Each measurement involved recording the following parameters: maximal radial displacement (Dm; $\mathrm{mm}$ ); contraction time (Tc; $\mathrm{ms}$ ): $\mathrm{Tc}$ as the time from $10 \%$ to $90 \%$ of $\mathrm{Dm}$; delay time (Td; ms) as the time from onset to $10 \%$ of Dm sides of the curve; sustained time (Ts; $\mathrm{ms}$ ) as the time between $50 \%$ of Dm on both the ascending and descending sides of the curve; and Half relaxation time ( $\mathrm{Tr}$ ) was the time from $90 \% \mathrm{Dm}$ to decline to $50 \%$ of the Dm in the relaxation phase ${ }^{27}$ (Fig. 1).

Statistical analyses. Normal distribution of all variables was determined by Kolmogorov-Smirnov test, together with the Lilliefors test, in order to verify that the sample distribution was normal, linear and homoscedastic. Relative reliability was quantified by the intraclass correlation coefficient (ICC), along with a 95\% confidence interval (CI), respect to the two measurements taken in each participant in all conditions. The ICC was calculated using a two-way-mixed effects and absolute agreement model ${ }^{28}$. ICC values under 0.50 were rated as low reliability, values between 0.50 and 0.75 indicates moderate reliability, values between 0.75 and 0.90 express good reliability and values greater than 0.90 indicates excellent reliability. Absolute reliability indices were expressed through the standard error of the mean (SEM, SEM\%), minimum detectable change (MDC, \%MDC) and coefficient of variation (CV) along with the respective $95 \%$ CI. The CV was calculated for raw data after being $\log$-transformed ${ }^{29}$. A CV $>10 \%$ was interpreted as insufficient absolute reliability ${ }^{17,30,31}$. The SEM is an index for 


\begin{tabular}{|c|c|c|c|c|c|c|c|c|c|c|c|c|c|c|c|c|c|c|}
\hline \multirow[b]{3}{*}{ VM } & \multicolumn{18}{|l|}{ Electrode $3.2 \mathrm{~cm}$} \\
\hline & \multirow[b]{2}{*}{$\mathrm{M} \pm \mathrm{SD}$} & \multicolumn{5}{|c|}{ Stimulus length 0.2} & \multirow[b]{2}{*}{$\mathrm{M} \pm \mathrm{SD}$} & \multicolumn{5}{|c|}{ Stimulus length 0.5} & \multirow[b]{2}{*}{$\mathbf{M} \pm \mathbf{S D}$} & \multicolumn{5}{|c|}{ Stimulus length 1} \\
\hline & & $\begin{array}{l}\text { ICC CI } \\
95 \%\end{array}$ & CV\% & $\begin{array}{l}\text { SEM } \\
\text { SEM\% }\end{array}$ & MDC & MDC\% & & $\begin{array}{l}\text { ICC CI } \\
95 \%\end{array}$ & $\mathrm{CV} \%$ & $\begin{array}{l}\text { SEM } \\
\text { SEM\% }\end{array}$ & MDC & MDC\% & & $\begin{array}{l}\text { ICC CI } \\
95 \%\end{array}$ & CV\% & $\begin{array}{l}\text { SEM } \\
\text { SEM\% }\end{array}$ & MDC & MDC\% \\
\hline $\begin{array}{l}\mathrm{Dm} \\
(\mathrm{mm})\end{array}$ & $8.18 \pm 1.50$ & $\begin{array}{l}0.97(0.95- \\
0.98)\end{array}$ & 3.0 & $\begin{array}{l}0.24 \pm \\
2.95\end{array}$ & 0.67 & 8.22 & $\begin{array}{l}8.50 \pm \\
1.51\end{array}$ & $\begin{array}{l}0.97 \\
(0.95- \\
0.98)\end{array}$ & 3.2 & $\begin{array}{l}0.26 \pm \\
3.10\end{array}$ & 0.73 & 8.62 & $\begin{array}{l}8.60 \pm \\
1.36\end{array}$ & $\begin{array}{l}0.97(0.95- \\
0.98)\end{array}$ & 3.1 & $\begin{array}{l}0.23 \pm \\
2.74\end{array}$ & 0.65 & 7.62 \\
\hline $\mathrm{Tc}(\mathrm{ms})$ & $22.52 \pm 2.10$ & $\begin{array}{l}0.95(0.91- \\
0.97)\end{array}$ & 2.2 & $\begin{array}{l}0.49 \pm \\
2.19\end{array}$ & 1.37 & 6.09 & $\begin{array}{l}22.17 \pm \\
2.04\end{array}$ & $\begin{array}{l}0.95 \\
(0.91- \\
0.97)\end{array}$ & 2.3 & $\begin{array}{l}0.47 \pm \\
2.11\end{array}$ & 1.30 & 5.87 & $\begin{array}{l}22.21 \pm \\
1.94\end{array}$ & $\begin{array}{l}0.95(0.90- \\
0.97)\end{array}$ & 2.2 & $\begin{array}{l}0.43 \pm \\
1.97\end{array}$ & 1.21 & 5.46 \\
\hline $\mathrm{Td}(\mathrm{ms})$ & $22.38 \pm 1.25$ & $\begin{array}{l}0.85(0.75- \\
0.91)\end{array}$ & 2.3 & $\begin{array}{l}0.50 \pm \\
2.23\end{array}$ & 1.39 & 6.21 & $\begin{array}{l}22.23 \pm \\
1.15\end{array}$ & $\begin{array}{l}0.88 \\
(0.81- \\
0.94)\end{array}$ & 1.9 & $\begin{array}{l}0.39 \pm \\
1.77\end{array}$ & 1.09 & 4.92 & $\begin{array}{l}22.24 \pm \\
1.21\end{array}$ & $\begin{array}{l}0.87(0.79- \\
0.93)\end{array}$ & 2.2 & $\begin{array}{l}0.45 \pm \\
2.02\end{array}$ & 1.25 & 5.62 \\
\hline $\operatorname{Tr}(\mathrm{ms})$ & $67.10 \pm 39.49$ & $\begin{array}{l}0.76(0.62- \\
0.86)\end{array}$ & 23.1 & $\begin{array}{l}21.25 \pm \\
31.66\end{array}$ & 58.92 & 87.80 & $\begin{array}{l}75.95 \pm \\
47.31\end{array}$ & $\begin{array}{l}0.85 \\
(0.75- \\
0.91)\end{array}$ & 28.7 & $\begin{array}{l}19.77 \pm \\
26.03\end{array}$ & 54.81 & 72.16 & $\begin{array}{l}71.09 \pm \\
43.61\end{array}$ & $\begin{array}{l}0.76(0.62- \\
0.86)\end{array}$ & 36.6 & $\begin{array}{l}23.69 \pm \\
33.32\end{array}$ & 65.66 & 92.37 \\
\hline \multirow[t]{2}{*}{ Ts (ms) } & $168.60 \pm 28.60$ & $\begin{array}{l}0.92(0.87- \\
0.96)\end{array}$ & 4.4 & $\begin{array}{l}8.10 \pm \\
4.80\end{array}$ & 22.46 & 13.32 & $\begin{array}{l}180.5 \pm \\
27.96\end{array}$ & $\begin{array}{l}0.91 \\
(0.86- \\
0.95)\end{array}$ & 6.2 & $\begin{array}{l}8.29 \pm \\
4.59\end{array}$ & 23.00 & 12.74 & $\begin{array}{l}175.6 \pm \\
26.84\end{array}$ & $\begin{array}{l}0.90(0.83- \\
0.94)\end{array}$ & 5.4 & $\begin{array}{l}9.38 \pm \\
5.34\end{array}$ & 26.01 & 14.81 \\
\hline & \multicolumn{18}{|l|}{ Electrode $5 \mathrm{~cm}$} \\
\hline $\begin{array}{l}\mathrm{Dm} \\
(\mathrm{mm})\end{array}$ & $8.65 \pm 1.34$ & $\begin{array}{l}0.97(0.94- \\
0.98)\end{array}$ & 3.0 & $\begin{array}{l}0.25 \pm \\
2.89\end{array}$ & 0.69 & 8.04 & $\begin{array}{l}8.67 \pm \\
1.36\end{array}$ & $\begin{array}{l}0.96 \\
(0.93- \\
0.98)\end{array}$ & 2.9 & $\begin{array}{l}0.31 \pm \\
3.65\end{array}$ & 0.88 & 10.16 & $\begin{array}{l}8.67 \pm \\
1.30\end{array}$ & $\begin{array}{l}0.95(0.91- \\
0.97)\end{array}$ & 3.8 & $\begin{array}{l}0.26 \pm \\
3.06\end{array}$ & 0.73 & 8.51 \\
\hline $\mathrm{Tc}(\mathrm{ms})$ & $21.90 \pm 1.98$ & $\begin{array}{l}0.97(0.95- \\
0.98)\end{array}$ & 1.6 & $\begin{array}{l}0.33 \pm \\
1.53\end{array}$ & 0.93 & 4.25 & $\begin{array}{l}21.50 \pm \\
1.97\end{array}$ & $\begin{array}{l}0.95 \\
(0.92- \\
0.97)\end{array}$ & 2.0 & $\begin{array}{l}0.43 \pm \\
2.01\end{array}$ & 1.20 & 5.60 & $\begin{array}{l}21.77 \pm \\
2.01\end{array}$ & $\begin{array}{l}0.95(0.91- \\
0.97)\end{array}$ & 2.4 & $\begin{array}{l}0.47 \pm \\
2.16\end{array}$ & 1.30 & 5.99 \\
\hline $\mathrm{Td}(\mathrm{ms})$ & $22.21 \pm 1.22$ & $\begin{array}{l}0.93(0.88- \\
0.96)\end{array}$ & 1.6 & $\begin{array}{l}0.33 \pm \\
1.49\end{array}$ & 0.92 & 4.15 & $\begin{array}{l}22.08 \pm \\
1.22\end{array}$ & $\begin{array}{l}0.90 \\
(0.83- \\
0.94)\end{array}$ & 1.6 & $\begin{array}{l}0.40 \pm \\
1.84\end{array}$ & 1.12 & 5.11 & $\begin{array}{l}22.06 \pm \\
1.09\end{array}$ & $\begin{array}{l}0.88(0.81- \\
0.93)\end{array}$ & 1.7 & $\begin{array}{l}0.39 \pm \\
1.78\end{array}$ & 1.09 & 4.96 \\
\hline $\operatorname{Tr}(\mathrm{ms})$ & $81.93 \pm 47.42$ & $\begin{array}{l}0.85(0.76- \\
0.91)\end{array}$ & 38.1 & $\begin{array}{l}22.69 \pm \\
27.69\end{array}$ & 62.90 & 76.77 & $\begin{array}{l}75.37 \pm \\
48.70\end{array}$ & $\begin{array}{l}0.89 \\
(0.81- \\
0.94)\end{array}$ & 25.8 & $\begin{array}{l}19.62 \pm \\
26.03\end{array}$ & 54.38 & 72.15 & $\begin{array}{l}62.76 \pm \\
40.17\end{array}$ & $\begin{array}{l}0.80(0.68- \\
0.88)\end{array}$ & 34.1 & $\begin{array}{l}21.77 \pm \\
34.68\end{array}$ & 60.34 & 96.15 \\
\hline $\mathrm{Ts}(\mathrm{ms})$ & $175.41 \pm 28.64$ & $\begin{array}{l}0.88(0.81- \\
0.93)\end{array}$ & 6.6 & $\begin{array}{l}10.61 \pm \\
6.04\end{array}$ & 29.42 & 16.77 & $\begin{array}{l}182.65 \\
\pm 30.24\end{array}$ & $\begin{array}{l}0.89 \\
(0.81- \\
0.94)\end{array}$ & 5.5 & $\begin{array}{l}8.36 \pm \\
4.58\end{array}$ & 23.19 & 12.69 & $\begin{array}{l}182.58 \\
\pm 28.43\end{array}$ & $\begin{array}{l}0.90(0.84- \\
0.95)\end{array}$ & 5.7 & $\begin{array}{l}9.09 \pm \\
4.98\end{array}$ & 25.20 & 13.80 \\
\hline
\end{tabular}

Table 2. Reliability in tensiomyography parameters for Vastus Medialis muscle $(n=36)$. Data are mean \pm SD. VM: Vastus Medialis; Dm: displacement; Tc: contraction time; Td: delay time; Tr: half-relaxation time; Ts: sustain time; ms: milliseconds. CV: coefficient of variation; $\mathrm{cm}$ : centimeters; ICC: intraclass correlation coefficient; $\mathrm{CI}$ : confidence interval; SEM standard error of measurement; MDC: minimum detectable change.

the precision of a measure and reflects the scores consistency within individual subjects ${ }^{31}$. The calculation of this index was made according Weir ${ }^{32}$ as follows equation: $\left(\mathrm{SEM}=\sqrt{ } \mathrm{MS}_{\mathrm{E}}\right)$, where $\mathrm{MS}_{\mathrm{E}}$ is the mean square error term from the repeated measures ANOVA. The \%SEM, which represents the relative amount of measurement error, was calculated according to Wagner et al. ${ }^{33}$ as follows equation: (SEM\% $=\mathrm{SEM} / M \times 100$ ), being $M$ the mean of the three TMG measurements. The MDC was calculated as equation: $(\mathrm{SEM} \times 1.96 \times \sqrt{ } 2)^{32}$. MDC\% was expressed as equation: $(\mathrm{MDC} / M \times 100)^{30}$, where $M$ is the mean of the three TMG measurements. To analyse the effect of the electrode size (i.e., 3.2 vs. $5 \mathrm{~cm}$ ) and the stimulus length (i.e., $0.2,0.5$ and $1 \mathrm{~ms}$ ), an analysis of the variance of repeated measures $(2 \times 3$ ANOVA $)$ was used. Bonferroni post hoc test with adjustment for $95 \%$ confidence interval was used to compare the main effects and identify significant individual differences. The effect sizes in repeated measures ANOVA were reported as partial eta square $\left(\eta_{p}^{2}\right)$ and interpreted as small $(0.01)$, moderate $(0.06)$, or large $(0.14)^{34}$. An alpha level of $\mathrm{p}<0.05$ was considered statistically significant. All data were analysed using SPSS v24.0 for Windows (SPSS Inc., Chicago, IL, USA).

\section{Results}

$\mathrm{Dm}$ and Tc parameters showed an excellent relative and absolute reliability with both electrode sizes and with all intensities for both muscles (Tables 2 and 3). Similarly, Td exhibited an excellent relative and absolute reliability in all conditions, except for the $3.2 \mathrm{~cm}$ electrode applied to the VM where the relative reliability was good. Ts showed a good to excellent relative reliability in both muscles, together with a good absolute reliability in the VM in all conditions. However, Ts exhibited an insufficient absolute reliability in the RF for all the conditions. Lastly, $\mathrm{Tr}$ showed insufficient absolute reliability for all the conditions for both muscles and a moderate to excellent relative reliability for both muscles. Based on the reliability results, Tr and Ts parameters were omitted in the ANOVA due to the insufficient absolute reliability.

As observed in Table 4, the stimulus length and the electrode size modulated the Tc of the VM with a large effect size $\left(\eta_{\mathrm{p}}^{2}=0.163-0.438\right)$. However, none of the conditions modulated the Tc of the RF. In the VM, a smaller electrode size caused a longer Tc response ( $22.30 \mathrm{vs} 21.72 \mathrm{~ms} ; 2.6 \%, \mathrm{p}=0.001$ for $3.2 \mathrm{vs} .5 \mathrm{~cm}$, respectively). In addition, a shorter stimulus length caused a longer Tc between 0.2 and $0.5 \mathrm{~ms}(22.21 \mathrm{vs} 21.83 \mathrm{~ms} ; 1.7 \%, \mathrm{p}=0.001)$. However, there were no differences in the Tc between stimuli neither of 0.5 and $1 \mathrm{~ms}$ nor between 0.2 and $1 \mathrm{~ms}$. 


\begin{tabular}{|c|c|c|c|c|c|c|c|c|c|c|c|c|c|c|c|c|c|c|}
\hline \multirow[b]{3}{*}{ RF } & \multicolumn{18}{|c|}{ Electrode $3.2 \mathrm{~cm}$} \\
\hline & \multirow[b]{2}{*}{$\mathbf{M} \pm \mathbf{S D}$} & \multicolumn{5}{|c|}{ Stimulus length 0.2} & \multirow[b]{2}{*}{$\mathrm{M} \pm \mathrm{SD}$} & \multicolumn{5}{|c|}{ Stimulus length 0.5} & \multirow[b]{2}{*}{$\mathrm{M} \pm \mathrm{SD}$} & \multicolumn{5}{|c|}{ Stimulus length 1} \\
\hline & & $\begin{array}{l}\text { ICC CI } \\
95 \%\end{array}$ & $\mathrm{CV} \%$ & $\begin{array}{l}\text { SEM } \\
\text { SEM\% }\end{array}$ & MDC & MDC\% & & ICC CI 95\% & $\mathrm{CV} \%$ & $\begin{array}{l}\text { SEM } \\
\text { SEM\% }\end{array}$ & MDC & MDC\% & & ICC CI 95\% & $\mathrm{CV} \%$ & $\begin{array}{l}\text { SEM } \\
\text { SEM\% }\end{array}$ & MDC & MDC $\%$ \\
\hline $\begin{array}{l}\mathrm{Dm} \\
(\mathrm{mm})\end{array}$ & $7.63 \pm 2.10$ & $\begin{array}{l}0.98(0.97- \\
0.99)\end{array}$ & 4.1 & $\begin{array}{l}0.28 \pm \\
3.77\end{array}$ & 0.79 & 10.46 & $\begin{array}{l}8.60 \pm \\
2.51\end{array}$ & $\begin{array}{l}0.98(0.97- \\
0.99)\end{array}$ & 3.7 & $\begin{array}{l}0.31 \pm \\
3.68\end{array}$ & 0.88 & 10.23 & $\begin{array}{l}9.23 \pm \\
2.19\end{array}$ & $\begin{array}{l}0.98(0.97- \\
0.99)\end{array}$ & 2.9 & $\begin{array}{l}0.28 \pm \\
3.09\end{array}$ & 0.79 & 8.59 \\
\hline $\mathrm{Tc}(\mathrm{ms})$ & $26.14 \pm 4.79$ & $\begin{array}{l}0.98(0.96- \\
0.99)\end{array}$ & 3.0 & $\begin{array}{l}0.67 \pm \\
2.58\end{array}$ & 1.87 & 7.15 & $\begin{array}{l}26.38 \pm \\
4.77\end{array}$ & $\begin{array}{l}0.98(0.97- \\
0.99)\end{array}$ & 2.4 & $\begin{array}{l}0.57 \pm \\
2.16\end{array}$ & 1.58 & 6.00 & $\begin{array}{l}26.95 \pm \\
4.67\end{array}$ & $\begin{array}{l}0.98(0.97- \\
0.99)\end{array}$ & 2.4 & $\begin{array}{l}0.57 \pm \\
2.12\end{array}$ & 1.59 & 5.90 \\
\hline $\mathrm{Td}(\mathrm{ms})$ & $24.50 \pm 1.96$ & $\begin{array}{l}0.94(0.90- \\
0.97)\end{array}$ & 2.3 & $\begin{array}{l}0.49 \pm \\
2.01\end{array}$ & 1.35 & 5.58 & $\begin{array}{l}24.82 \pm \\
1.90\end{array}$ & $\begin{array}{l}0.96(0.93- \\
0.98)\end{array}$ & 1.8 & $\begin{array}{l}0.40 \pm \\
1.61\end{array}$ & 1.08 & 4.46 & $\begin{array}{l}24.95 \pm \\
1.93\end{array}$ & $\begin{array}{l}0.93(0.88- \\
0.96)\end{array}$ & 2.7 & $\begin{array}{l}0.53 \pm \\
2.14\end{array}$ & 1.48 & 5.96 \\
\hline $\operatorname{Tr}(\mathrm{ms})$ & $\begin{array}{l}73.77 \pm \\
39.12\end{array}$ & $\begin{array}{l}0.86(0.77- \\
0.92)\end{array}$ & 26.2 & $\begin{array}{l}14.92 \pm \\
20.23\end{array}$ & 41.37 & 56.09 & $\begin{array}{l}65.21 \pm \\
40.77\end{array}$ & $\begin{array}{l}0.90(0.84- \\
0.95)\end{array}$ & 31.1 & $\begin{array}{l}13.26 \pm \\
20.34\end{array}$ & 36.77 & 56.38 & $\begin{array}{l}73.52 \pm \\
52.98\end{array}$ & $\begin{array}{l}0.96(0.92- \\
0.97)\end{array}$ & 18.8 & $\begin{array}{l}12.00 \pm \\
16.32\end{array}$ & 33.26 & 45.24 \\
\hline \multirow[t]{2}{*}{$\mathrm{Ts}(\mathrm{ms})$} & $\begin{array}{l}117.77 \pm \\
44.88\end{array}$ & $\begin{array}{l}0.87(0.78- \\
0.92)\end{array}$ & 21.2 & $\begin{array}{l}16.70 \pm \\
14.18\end{array}$ & 46.29 & 39.35 & $\begin{array}{l}105.07 \\
\pm 44.86\end{array}$ & $\begin{array}{l}0.91(0.86- \\
0.95)\end{array}$ & 18.2 & $\begin{array}{l}13.66 \pm \\
13.00\end{array}$ & 37.87 & 36.04 & $\begin{array}{l}109.30 \\
\pm 52.25\end{array}$ & $\begin{array}{l}0.96(0.92- \\
0.97)\end{array}$ & 12.7 & $\begin{array}{l}12.54 \pm \\
11.47\end{array}$ & 34.77 & 31.79 \\
\hline & \multicolumn{18}{|c|}{ Electrode $5 \mathrm{~cm}$} \\
\hline $\begin{array}{l}\mathrm{Dm} \\
(\mathrm{mm})\end{array}$ & $8.67 \pm 2.24$ & $\begin{array}{l}0.98(0.97- \\
0.99)\end{array}$ & 4.2 & $\begin{array}{l}0.35 \pm \\
4.10\end{array}$ & 0.98 & 11.39 & $\begin{array}{l}9.53 \pm \\
2.16\end{array}$ & $\begin{array}{l}0.98(0.96- \\
0.99)\end{array}$ & 4.2 & $\begin{array}{l}0.31 \pm \\
3.29\end{array}$ & 0.87 & 9.15 & $\begin{array}{l}10.04 \pm \\
2.23\end{array}$ & $\begin{array}{l}0.98(0.96- \\
0.99)\end{array}$ & 3.5 & $\begin{array}{l}0.33 \pm \\
3.37\end{array}$ & 0.93 & 9.36 \\
\hline $\mathrm{Tc}(\mathrm{ms})$ & $26.25 \pm 4.81$ & $\begin{array}{l}0.97(0.95- \\
0.98)\end{array}$ & 2.7 & $\begin{array}{l}0.87 \pm \\
3.33\end{array}$ & 2.42 & 9.24 & $\begin{array}{l}26.19 \pm \\
4.22\end{array}$ & $\begin{array}{l}0.98(0.96- \\
0.99)\end{array}$ & 2.6 & $\begin{array}{l}0.61 \pm \\
2.34\end{array}$ & 1.70 & 6.50 & $\begin{array}{l}26.30 \pm \\
4.12\end{array}$ & $\begin{array}{l}0.99(0.98- \\
0.99)\end{array}$ & 2.0 & $\begin{array}{l}0.45 \pm \\
1.72\end{array}$ & 1.26 & 4.79 \\
\hline $\mathrm{Td}(\mathrm{ms})$ & $24.79 \pm 1.96$ & $\begin{array}{l}0.93(0.84- \\
0.96)\end{array}$ & 2.0 & $\begin{array}{l}0.53 \pm \\
2.15\end{array}$ & 1.47 & 5.96 & $\begin{array}{l}25.14 \pm \\
1.77\end{array}$ & $\begin{array}{l}0.94(0.89- \\
0.96)\end{array}$ & 2.0 & $\begin{array}{l}0.45 \pm \\
1.80\end{array}$ & 1.25 & 5.00 & $\begin{array}{l}25.36 \pm \\
1.87\end{array}$ & $\begin{array}{l}0.94(0.90- \\
0.97)\end{array}$ & 2.3 & $\begin{array}{l}0.46 \pm \\
1.81\end{array}$ & 1.27 & 5.04 \\
\hline $\operatorname{Tr}(\mathrm{ms})$ & $\begin{array}{l}75.64 \pm \\
49.14\end{array}$ & $\begin{array}{l}0.83(0.73- \\
0.90)\end{array}$ & 33.0 & $\begin{array}{l}23.37 \pm \\
30.89\end{array}$ & 64.79 & 85.66 & $\begin{array}{l}69.34 \pm \\
53.41\end{array}$ & $\begin{array}{l}0.82(0.71- \\
0.90)\end{array}$ & 40.9 & $\begin{array}{l}28.75 \pm \\
41.46\end{array}$ & 79.70 & 114.94 & $\begin{array}{l}64.96 \pm \\
51.58\end{array}$ & $\begin{array}{l}0.94(0.89- \\
0.96)\end{array}$ & 22 & $\begin{array}{l}19.68 \pm \\
30.29\end{array}$ & 46.26 & 71.21 \\
\hline Ts (ms) & $\begin{array}{l}110.95 \pm \\
45.82\end{array}$ & $\begin{array}{l}0.87(0.79- \\
0.93)\end{array}$ & 20.3 & $\begin{array}{l}17.10 \pm \\
15.41\end{array}$ & 47.40 & 42.72 & $\begin{array}{l}102.17 \\
\pm 52.92\end{array}$ & $\begin{array}{l}0.83(0.73- \\
0.90)\end{array}$ & 22.9 & $\begin{array}{l}26.90 \pm \\
26.32\end{array}$ & 74.56 & 72.98 & $\begin{array}{l}97.92 \pm \\
51.98\end{array}$ & $\begin{array}{l}0.94(0.89- \\
0.96)\end{array}$ & 14.2 & $\begin{array}{l}16.94 \pm \\
17.29\end{array}$ & 46.97 & 47.97 \\
\hline
\end{tabular}

Table 3. Reliability in tensiomyography parameters for Rectus Femoris muscle $(n=36)$. Data are mean \pm SD. RF: Rectus Femoris; Dm: displacement; Tc: contraction time; Td: delay time; Tr: half-relaxation time; Ts: sustain time; ms: milliseconds. CV: coefficient of variation; $\mathrm{cm}$ : centimeters; ICC: intraclass correlation coefficient; CI: confidence interval; SEM standard error of measurement; MDC: minimum detectable change.

The Dm of the VM moderately increased $\left(\eta_{p}^{2}=0.089\right)$ as the stimulus length increased, although only significantly between the duration of 0.2 and $0.5 \mathrm{~ms}(8.41 \mathrm{vs} 8.58 \mathrm{~mm}, 2.0 \%, \mathrm{p}=0.025)$ (Fig. 2$)$. The Dm of the RF also increased as the stimulus length increased with a large effect size $\left(\eta_{p}^{2}=0.606\right)$ (Fig. 3). In addition, it also increased $10.4 \%(\mathrm{p}=0.001)$ between 0.2 and $0.5 \mathrm{~ms}$ duration, $7.0 \%(\mathrm{p}=0.001)$ between 0.5 and $1 \mathrm{~ms}$ and $18.2 \%$ $(\mathrm{p}=0.001)$ between 0.2 and $1 \mathrm{~ms}$. The use of the largest electrode $(5 \mathrm{~cm})$ caused a greater Dm than using the smaller one $(3.2 \mathrm{~cm})$ in both the $\operatorname{VM}(2.8 \%, \mathrm{p}=0.036)$ and $\mathrm{RF}(10.8 \%, \mathrm{p}=0.001)$. In addition, the interaction stimulus length $\mathrm{x}$ electrode size moderately $\left(\eta_{\mathrm{p}}^{2}=0.117\right)$ modulated the Dm of the VM, which occurred with all stimulus length $(8.18-8.65 \mathrm{~mm}$ at $0.2 \mathrm{~ms} ; 8.50-8.67 \mathrm{~mm}$ at $0.5 \mathrm{~ms} ; 8.60-8.67 \mathrm{~mm}$ at $1 \mathrm{~ms} ; \mathrm{p}=0.013)$.

The Td of the VM was only significantly affected by the size of the electrode. The use of a larger electrode $(5 \mathrm{~cm})$ caused a lower Td $(22.28 \mathrm{vs} 22.11 \mathrm{~ms}, 0.7 \%, \mathrm{p}=0.023)$. However, the Td of the RF increased as the stimulus length increases with a large effect size $\left(\eta_{p}^{2}=0.237\right)$. It increased by $1.4 \%(p=0.006)$ between 0.2 and $0.5 \mathrm{~ms}$ duration, $0.7 \%(\mathrm{p}=0.036)$ between 0.5 and $1 \mathrm{~ms}$ and $2.1 \%(\mathrm{p}=0.001)$ between 0.2 and $1 \mathrm{~ms}$. In addition, the electrode size also modulated the Td of the RF with a large effect size $\left(\eta_{p}^{2}=0.327\right)$, so that the use of the largest electrode $(5 \mathrm{~cm})$ caused longer $\mathrm{Td}(1.4 \%, \mathrm{p}=0.001)$.

\section{Discussion}

Summarizing, the results of this study show that the stimulus length (duration of the TNS pulse) applied to the RF and VM muscles and the size of the electrode significantly influence the muscle's contractile properties as measured by a single-twitch TMG technique. The main finding is that Dm increases significantly both, as the duration of the TNS increases and with the use of a larger electrode $(5 \mathrm{~cm})$ in both muscles. However, Tc and Td are less affected by both conditions and not entirely clear.

Our results are consistent with previous reviews on the topic showing that Tr parameter should not be used in research or clinical environments due to their insufficient absolute reliability and high measurement error ${ }^{2,16}$. Similarly, Ts parameter should not be used in these areas since it has shown a CV higher than $10 \%{ }^{17}$. On the contrary, Dm, Tc and Td parameters have shown a high relative reliability in all muscles and situations evaluated, which is in line with the findings of Lohr et al. ${ }^{35}$ but also an absolute reliability through a low CV, \%SEM and \%MDC, as opposed to the results of Lohr et al..$^{35}$ which indicates a discordant absolute reliability in the Dm and moderate positive in the Tc. Hence, the effect of stimulus length and electrode size on Tr and Ts was not examined, since these parameters did not meet the reliability requirements.

The electrical energy available to stimulate the muscle is influenced by the duration of the applied electrical impulse as shown by the equation ${ }^{20}:(E=V x I x \Delta t)$, where $\mathrm{E}$ is the electrical energy, $\mathrm{V}$ are volts, I equals current and $\Delta t$ is the duration of the TNS pulse. It appears that, at stimulus length below $1 \mathrm{~ms}$, there is insufficient electrical energy available to maximally stimulate all motor units ${ }^{36}$. Moreover, given that smaller motor units are easier to stimulate than larger motor units ${ }^{37,38}$, shorter stimulus length appear to favor type I slow twitch muscle fibers over type II fast twitch muscle fibers, which are more difficult to activate ${ }^{36}$. Our results match those found 


\begin{tabular}{|c|c|c|c|c|c|}
\hline & EFFECT & $\mathbf{F}$ & Df & $\mathbf{P}$ & $\eta^{2}$ \\
\hline \multirow{3}{*}{$\mathrm{Tc} V M$} & stimulus length* & 6.812 & 2 & 0.002 & 0.163 \\
\hline & electrode size $*$ & 27.244 & 1 & 0.001 & 0.438 \\
\hline & stimulus length $\times$ electrode size & 1.341 & 2 & 0.268 & 0.037 \\
\hline \multirow{3}{*}{ Tc RF } & stimulus length & 1.526 & 2 & 0.225 & 0.042 \\
\hline & electrode size & 0.836 & 1 & 0.367 & 0.023 \\
\hline & stimulus length $\times$ electrode size & 1.774 & 2 & 0.177 & 0.048 \\
\hline \multirow{3}{*}{ Dm VM } & stimulus length* & 3.418 & 2 & 0.038 & 0.089 \\
\hline & electrode size $*$ & 4.757 & 1 & 0.036 & 0.120 \\
\hline & stimulus length $\times$ electrode size $*$ & 4.628 & 2 & 0.013 & 0.117 \\
\hline \multirow{3}{*}{$\mathrm{Dm} R F$} & stimulus length* & 53.793 & 2 & 0.001 & 0.606 \\
\hline & electrode size $*$ & 49.926 & 1 & 0.001 & 0.588 \\
\hline & stimulus length $\times$ electrode size & 0.675 & 2 & 0.513 & 0.019 \\
\hline \multirow{3}{*}{ Td VM } & stimulus length & 2.041 & 2 & 0.138 & 0.055 \\
\hline & electrode size $*$ & 5.694 & 1 & 0.023 & 0.140 \\
\hline & stimulus length $\times$ electrode size & 0.082 & 2 & 0.922 & 0.002 \\
\hline \multirow{3}{*}{ Td RF } & stimulus length* & 10.865 & 2 & 0.001 & 0.237 \\
\hline & electrode size $*$ & 17.002 & 1 & 0.001 & 0.327 \\
\hline & stimulus length $\times$ electrode size & 0.386 & 2 & 0.681 & 0.011 \\
\hline
\end{tabular}

Table 4. Effects of different stimulus length and electrode size on different tensiomography parameters. Interactions between stimulus length $(0.2,0.5$ and $1 \mathrm{~ms})$, and electrode size $(3.2 \mathrm{vs} .5 \mathrm{~cm}) .{ }^{*} \mathrm{p}<0.05$.

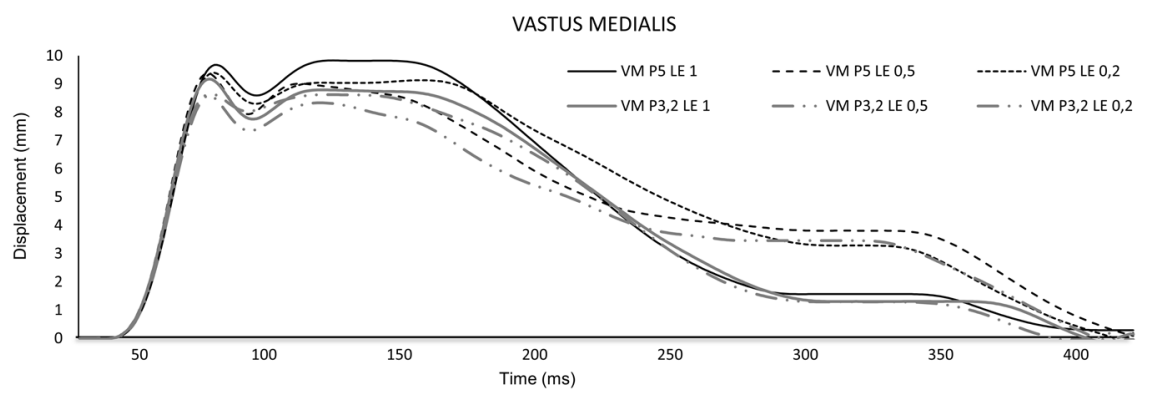

Figure 2. Displacement-time curves of Vastus Medialis of the dominant leg, according to the electrode size $(3.2-5 \mathrm{~cm})$ and the stimulus length $(0.2,0.5$, and $1 \mathrm{~ms})$.

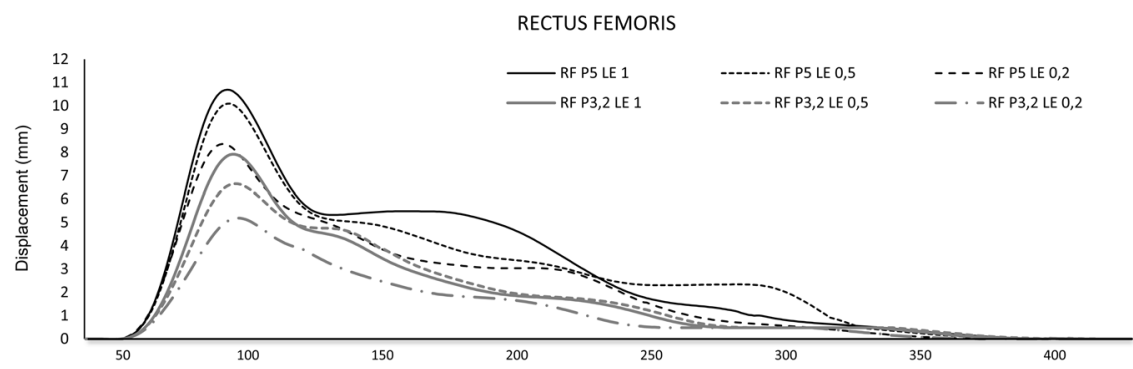

Figure 3. Displacement-time curves of Rectus Femoris of the dominant leg, according to the electrode size $(3.2-5 \mathrm{~cm})$ and the stimulus length $(0.2,0.5$, and $1 \mathrm{~ms})$.

by McAndrew et al. ${ }^{14}$, who reported that stimulus length above $0.3 \mathrm{~ms}$ provide both a maximal lateral displacement of the muscle's belly and stable measures of its contractile properties. Although the previous authors used a laser-based MMG technique, their results can be extrapolated to ours since it has been demonstrated that both laser- and CDS shows good-to-excellent reliability for the assessment of muscle contractile properties with no significant differences between them ${ }^{15}$. In fact, it should be noted that there are data showing that the radial muscle displacement $(\mathrm{Dm})$ increases linearly with muscle torque up to $68 \%$ of maximal voluntary contraction ${ }^{6}$. This fact may suggest that, maintaining linearity, using shorter pulse duration $(0.2 \mathrm{~ms})$ could elicit low torque production, affecting to the wave-form and then to all the TMG parameters. 
To our knowledge, to date there has been no study that has analysed the effects of the electrodes size for muscle electrical stimulation measured by TMG. However, it has been previously analysed in transcutaneous electrical nerve stimulation (TENS) ${ }^{39}$. This author used 4 types of electrodes: $3 \times 3,6 \times 69 \times 9$ and $5 \times 16.2 \mathrm{~cm}$ on the quadriceps with the objective of examining the effects of electrode size at neural and motor level, reaching the conclusion that the larger the electrode, the greater the participation of motor units. Our results seem to be in agreement with those obtained by Alon ${ }^{39}$ with respect to the electrode size, since both VM and RF increased their Dm with the largest electrode. A reasonable explanation is that a larger electrode is able to recruit more motor units on the transverse axis and therefore greater amount of muscle mass displacement directly influences an increase in Dm. However, this hypothesis will still have to be proven.

On the other hand, the overall \%SEM and \%MDC results of both muscles seem to point out, in a general way, that increasing electrode size and pulse duration could increase the data accuracy and minimize the measurement error, although it would be necessary more research with other muscle groups to verify this point. A priori, to minimize measurement errors with TMG parameters, it should be recommended standardizing TMG protocols to develop a TMG standard operating procedure such that experimental studies may be comparable. In order to do this, based on the present findings and previous research, it is recommended: (1) to take into account the correct stimulus length (1 ms) to maximize Dm; (2) to use electrodes of $5 \mathrm{~cm}$; (3) to use specific guides for each muscle of inter-electrode distance ${ }^{18,19}$; and (4) additional recommendations for a correct measurement protocol are described in the review of Macgregor et al. ${ }^{2}$. In this line, Lohr et al. ${ }^{35}$ have indicated that it is necessary to have high methodological standards for conduct and reporting TMG studies, and these recommendations could be helpful for this purpose.

\section{Limitations, strengths and practical applications}

Finally, it is important pointing that, the maximal TNS voltage required to produce peak lateral displacement of a muscle's belly is unique to each muscle and is determined as the voltage that first produces an undistorted parabolic MMG waveform of maximal amplitude ${ }^{14}$. In this regard, we used a unique intensity ( $\left.100 \mathrm{~mA}\right)$ in our study to be able to homogenize and compare subjects and muscles with each other. This was done because the current and well accepted ramp protocol ${ }^{2,8}$, used to decide individual intensity would have made it difficult to compare results between subjects' muscles. This could have been solved by normalizing the values of each parameter of each subject/muscle to, for example, a maximum voluntary contraction. However, we did not have the necessary tools for this purpose, what is a limitation of this study. In addition, a duration of TNS above $1 \mathrm{~ms}$ could also have been used to try to stimulate larger motor units, but the TMG software did not allow this when the experiment was performed. Furthermore, it has only been evaluated two muscles, both of the lower limb, which may be another limitation of this study. On the other hand, the main strength of the present study is the sample size and the use of several set configurations for both electrode size and stimulus pulse duration, and as well as that the same evaluator with more of 7 years' experience took all measurements. In terms of practical applications, TMG researchers and practitioners can base their measurement protocol on the findings of this study. Our data indicates that a stimulation pulse duration of $1 \mathrm{~ms}$ together with the election of a $5 \times 5 \mathrm{~cm}$ electrode size is necessary to reach a reliable and reproducible assessment of both RF and VM muscles contractile properties. Conversely, the use of a smaller electrode or a stimulus length of less than $1 \mathrm{~ms}$ would be a risk to guarantee the reproducibility of the measurement taken with TMG.

\section{Conclusions}

The duration of the stimulus length applied to the RF and VM muscles and electrode size significantly influence the muscle's contractile properties as measured by a single-twitch TMG technique. In fact, the Dm increases substantially as the duration of the TNS increases and with the use of the larger electrode $(5 \mathrm{~cm})$ in both muscles. However, Tc and Td are less affected by both conditions and not entirely clear. Therefore this study indicates that a stimulus pulse duration of $1 \mathrm{~ms}$ together with the election of a $5 \times 5 \mathrm{~cm}$ electrode size is necessary to reach a reliable and reproducible assessment of both rectus and vastus medialis muscles contractile properties.

\section{Data availability}

The TMG recordings utilized in the current study will be uploaded to an open access server and will be available to anyone who would like to re-analyse them.

Received: 24 November 2019; Accepted: 27 April 2020;

Published online: 18 May 2020

\section{References}

1. Ibitoye, M. O., Hamzaid, N. A., Zuniga, J. M. \& Abdul Wahab, A. K. Mechanomyography and muscle function assessment: a review of current state and prospects. Clin Biomech (Bristol, Avon). 29, 691-704 (2014).

2. Macgregor, L. J., Hunter, A. M., Orizio, C., Fairweather, M. M. \& Ditroilo, M. Assessment of Skeletal Muscle Contractile Properties by Radial Displacement: The Case for Tensiomyography. Sport Med. 48, 1607-1620 (2018).

3. Simunič, B. et al. Noninvasive estimation of myosin heavy chain composition in human skeletal muscle. Med Sci Sports Exer. 43, 1619-1625 (2011).

4. Dahmane, R., Djordjevič, S., Šimunič, B. \& Valenčič, V. Spatial fiber type distribution in normal human muscle: Histochemical and tensiomyographical evaluation. J Biomech. 38, 2451-2459 (2005).

5. Zubac, D. \& Šimunič, B. Skeletal Muscle Contraction Time and Tone Decrease After 8 Weeks of Plyometric Training. J Strength Cond Res. 31, 1610-1619 (2017).

6. Pišot, R. et al. Whole muscle contractile parameters and thickness loss during 35-day bed rest. Eur J Appl Physiol. 104, 409-414 (2008).

7. Šimunič, B. Two-dimensional spatial error distribution of key tensiomyographic parameters. J Biomech. 92, 92-97 (2019).

8. García-García, O., Cuba-Dorado, A., Álvarez-Yates, T., Carballo-López, J. \& Iglesias-Caamaño, M. Clinical utility of tensiomyography for muscle function analysis in athletes. Open Access J Sports Med. 10, 49-69 (2019). 
9. Baraja-Vegas, L. et al. Localization of Muscle Edema and Changes on Muscle Contractility After Dry Needling of Latent Trigger Points in the Gastrocnemius Muscle. Pain Med. 20, 1387-1394 (2019).

10. Sánchez-Ureña, B., Rojas-Valverde, D. \& Gutiérrez-Vargas, R. Effectiveness of Two Cold Water Immersion Protocols on Neuromuscular Function Recovery: A Tensiomyography Study. Front Physiol. 9, 1-7 (2018).

11. Ginz, H. F., Zorzato, F., Iaizzo, P. A. \& Urwyler, A. Effect of three anaesthetic techniques on isometric skeletal muscle strength. $\mathrm{Br}$ JAnaesth. 92, 367-372 (2004)

12. Kimura, T., Hamada, T., Massako Ueno, L. \& Moritani, T. Changes in contractile properties and neuromuscular propagation evaluated by simultaneous mechanomyogram and electromyogram during experimentally induced hypothermia. J Electromyogr Kinesiol. 13, 433-440 (2003).

13. Maffiuletti, N. A. et al. Effect of electromyostimulation training on soleus and gastrocnemii H-and T-reflex properties. Eur J Appl Physiol. 90, 601-607 (2003).

14. McAndrew, D. J., Rosser, N. A. D., Mark, J. \& Brown, M. Mechanomyographic Measures of Muscle Contractile Properties are Influenced by the Duration of the Stimulatory Pulse. J Appl Res. 6, 142-152 (2006).

15. Seidl, L., Tosovic, D. \& Brown, J. M. Test-Retest Reliability and Reproducibility of Laser- versus Contact-Displacement Sensors in Mechanomyography: Implications for Musculoskeletal Research. J Appl Biomech. 33, 130-136 (2017).

16. Martín-Rodríguez, S., Loturco, I., Hunter, A. M., Rodríguez-Ruiz, D. \& Munguia-Izquierdo, D. Reliability and Measurement Error of Tensiomyography to Assess Mechanical Muscle Function. J Strength Cond Res. 31, 3524-3536 (2017).

17. Tous-Fajardo, J. et al. Inter-r.ater reliability of muscle contractile property measurements using non-invasive tensiomyography. $J$ Electromyogr Kinesiol. 20, 761-766 (2010).

18. Wilson, H. V., Johnson, M. I. \& Francis, P. Repeated stimulation, inter-stimulus interval and inter-electrode distance alters muscle contractile properties as measured by Tensiomyography. PloS One. 13, 1-15 (2018).

19. Wilson, H. V., Jones, A., Johnson, M. I. \& Francis, P. The effect of inter-electrode distance on radial muscle displacement and contraction time of the biceps femoris, gastrocnemius medialis and biceps brachii, using tensiomyography in healthy participants. Physiol Meas. 40(7), 075007, https://doi.org/10.1088/1361-6579/ab1cef (2019).

20. Alon., C., Kantor, G. \& Ho, H. S. Effects of Electrode Size on Basic Excitatory Responses and on Selected Stimulus Parameters. J Orthop Sports Phys. 20, 29-35 (1994).

21. Forrester, B. J. \& Petrofsky, J. S. Effect of electrode size, shape, and placement during electrical stimulation. J Appl Res. 4, 346-54 (2004).

22. Hermens, H. J., Freriks, B., Disselhorst-Klug, C. \& Rau, G. Development of recommendations for SEMG sensors and sensor placement procedures. J Electromyogr Kinesiol. 10, 361-374 (2000).

23. García-García, O., Serrano-Gómez, V., Hernández-Mendo, A. \& Tapia-Flores, A. Assessment of the in-season changes in mechanical and neuromuscular characteristics in professional soccer players. J Sports Med Phys Fitness. 56, 714-723 (2016).

24. García-Manso, J. M. et al. Effect of cold-water immersion on skeletal muscle contractile properties in soccer players. Am J Phys Med Rehabil. 90(5), 356-363 (2011).

25. García-Manso, J. M. et al. Effect of high-load and high-volume resistance exercise on the tensiomyographic twitch response of biceps brachii. J Electromyogr Kinesiol. 22(4), 612-619 (2012).

26. Rodríguez-Ruiz, D. et al. Analysis of the response speed of musculature of the knee in professional male and female volleyball players. BioMed research international. https://doi.org/10.1155/2014/239708 (2014).

27. Valencic, V. \& Knez, N. Measuring of skeletal muscles' dynamic properties. Artif Organs. 21, 240-242 (1997)

28. McGraw, K. O. \& Wong, S. P. Forming inferences about some intraclass correlation coefficients. Psychological Methods. 1, 30-46 (1996).

29. Hopkins, W. G. Measures of Reliability in Sports Medicine and Science. Sport Med. 30, 1-15 (2000).

30. Ditroilo, M., Smith, I. J., Fairweather, M. M. \& Hunter, A. M. Long-term stability of tensiomyography measured under different muscle conditions. J Electromyogr Kinesiol. 23, 558-563 (2013).

31. Lohr, C., Braumann, K. M., Reer, R., Schroeder, J. \& Schmidt, T. Reliability of tensiomyography and myotonometry in detecting mechanical and contractile characteristics of the lumbar erector spinae in healthy volunteers. Eur J Appl Physiol. 118, 1349-1359 (2018).

32. Weir, J. P. Quantifying Test-Retest Reliability Using the Intraclass Correlation Coefficient and the SEM. J Strength Cond Res. 19, 231-240 (2005).

33. Wagner, J. M., Rhodes, J. A. \& Patten, C. Reproducibility and minimal detectable change of three-dimensional kinematic analysis of reaching tasks in people with hemiparesis after stroke. Phys Ther. 88, 652-663 (2008).

34. Cohen, J. Statistical Power Analysis for the Behavioral Sciences (ed. Hillsdale, NJ: Lawrence Erlbaum, 2nd ed.) (1988).

35. Lohr, C. et al. Diagnostic accuracy, validity, and reliability of Tensiomyography to assess muscle function and exercise-induced fatigue in healthy participants. A systematic review with meta-analysis. J Electromyogr Kinesiol. 47, 65-87 (2019).

36. Bickel, C. S., Gregory, C. M. \& Dean, J. C. Motor unit recruitment during neuromuscular electrical stimulation: a critical appraisal. Eur J Appl Physiol. 111, 2399-2407 (2011).

37. Henneman, E., Somjen, G. \& Carpenter, D. O. Excitability and inhibitability of motoneurons of different sizes. J Neurophysiol. 28, 599-620 (1965)

38. Milner-Brown, H. S., Stein, R. B. \& Yemm, R. The orderly recruitment of human motor units during voluntary isometric contractions. J Physiol. 230, 359-370 (1973).

39. Alon, G. High Voltage Stimulation: Effects of Electrode Size on Basic Excitatory Responses. Phys Ther. 65, 890-895 (1985).

Author contributions

F.P.S., F.P.B., I.J.B. and O.G.G. conceived, coordinated, and designed the study. S.M.R. made suggestions that improved the design and wrote the manuscript together with F.P.S. in equal conditions. L.B.V., J.B.F. and F.P.S. carried out the experimental part. O.G.G. and I.J.B. analyzed data. F.P.B. and O.G.G. contributed to edit and review manuscript while helped in the drafting of the manuscript. All authors have read and approved the current manuscript.

\section{Competing interests}

The authors declare no competing interests.

Additional information

Correspondence and requests for materials should be addressed to Ó.G.-G.

Reprints and permissions information is available at www.nature.com/reprints.

Publisher's note Springer Nature remains neutral with regard to jurisdictional claims in published maps and institutional affiliations. 
(c) (i) Open Access This article is licensed under a Creative Commons Attribution 4.0 International License, which permits use, sharing, adaptation, distribution and reproduction in any medium or format, as long as you give appropriate credit to the original author(s) and the source, provide a link to the Creative Commons license, and indicate if changes were made. The images or other third party material in this article are included in the article's Creative Commons license, unless indicated otherwise in a credit line to the material. If material is not included in the article's Creative Commons license and your intended use is not permitted by statutory regulation or exceeds the permitted use, you will need to obtain permission directly from the copyright holder. To view a copy of this license, visit http://creativecommons.org/licenses/by/4.0/.

(C) The Author(s) 2020 